\title{
Tolerância a falhas em ambientes de computação em nuvem
}

\author{
Vinicius S. Andrade ${ }^{1}$, Aleardo Manacero ${ }^{1}$, Roberta Spolon ${ }^{2}$ Renata S. Lobato $^{1}$ \\ ${ }^{1}$ Depto. de Ciências de Computação e Estatística \\ Universidade Estadual Paulista - Unesp \\ São José do Rio Preto - SP - Brasil \\ ${ }^{2}$ Depto. de Computação \\ Universidade Estadual Paulista - Unesp \\ Bauru - SP - Brasil \\ viniciusarmani@gmail.com, aleardo@sjrp.unesp.br
}

\begin{abstract}
Cloud computing offers computer services over the internet using virtual machines. In clouds, there are several factors that can cause errors that lead to service failure. To avoid disruptions beyond the service failure itself, it is important to have a reliable fault tolerance support, ensuring that the environment recovers in its entirety, in an agile way. A manager of a cloud system may have difficulty chosing the best fault tolerance approach to his system because there are too many solutions suggested, aiming towards different goals. This work proposes a systematic approach to categorize such solutions through a survey about methods for fault tolerance in a cloud computing infrastructure.
\end{abstract}

Resumo. Computação em nuvem oferece serviços de computação pela internet, utilizando máquinas virtuais. Diversos fatores podem ocasionar erros que levem a falha(s) no serviço na nuvem. Para evitar transtornos, além da própria falha, é importante que o suporte de tolerância a falhas seja robusto, garantindo que o ambiente se recupere na íntegra, de maneira ágil. Um administrador de um sistema em nuvem pode ter dificuldade em escolher a melhor abordagem para tolerância a falhas em seu sistema entre as muitas soluções propostas, orientadas a diferentes objetivos. Aqui se propõe uma abordagem sistemática para categorizar tais soluções por meio de uma revisão sobre os métodos para tolerância a falhas em uma infraestrutura de computação em nuvem.

\section{Introdução}

O objetivo de computação em nuvem é oferecer serviços por meio de recursos distribuídos, que possam ser provisionados e liberados com mínimo esforço de gerenciamento. Isso implica em tratar possíveis falhas de forma a garantir que o atendimento ocorra com o mínimo possível de disrupções. Como falhas são inevitáveis é importante que se entenda como ocorrem e como podem ser evitadas ou corrigidas, implementando esse tratamento nos ambientes de gerenciamento da nuvem.

Apesar de contraditório, o fato de a literatura da área apresentar uma enorme quantidade de propostas de técnicas para o tratamento de falhas acaba sendo um problema. Isso ocorre pois tais técnicas apresentam abordagens e focos bastante distintos, assim, escolher aquela que melhor se adéqua ao ambiente específico de uma nuvem se torna complexo. O trabalho aqui apresentado faz uma primeira sistematização dessas contribuições, considerando o período do ano de 2010 a 2017. 


\section{Computação em nuvem}

A computação em nuvem é um termo abrangente para descrever uma categoria de serviços de computação sob demanda. Sua infraestrutura de computação é vista como uma "nuvem", em que empresas e indivíduos acessam aplicativos de qualquer lugar do mundo por meio da internet [Buyya et al. 2009]. Para que o modelo de computação em nuvem atenda às necessidades e expectativas dos consumidores é essencial que o serviço oferecido pela nuvam seja: self-service; de uso medido e faturado; elástico; e personalizável [Buyya et al. 2011].

\section{Tolerância a Falhas}

De acordo com Tanenbaum e Van Steen [Tanenbaum and Van Steen 2007] um defeito no sistema é ocasionado pela ocorrência de algum erro, criado pela ocorrência de uma falha. A identificação da falha e sua correção são aspectos importantes em qualquer sistema computacional, em especial em sistemas distribuídos como as nuvens.

Um sistema tolerante a falhas pode ser composto por uma ou mais técnicas, que se subdividem em reativas e proativas. A Tabela 1 apresenta alguns exemplos da aplicação de técnicas de tolerância a falhas em sistemas específicos [Bala and Chana 2012]. Nela é possível observar que mesmo situações semelhantes levam a soluções diversas, comprovando a dificuldade do processo de escolha de solução.

Tabela 1. Resumo das técnicas de tolerância a falhas para sistemas específicos.

\begin{tabular}{|l|l|l|l|l|l|l|}
\hline Sistema & $\begin{array}{l}\text { Técnica de } \\
\text { tolerância a } \\
\text { falha }\end{array}$ & Políticas & $\begin{array}{l}\text { Estrutura de } \\
\text { programação }\end{array}$ & Ambiente & $\begin{array}{l}\text { Tipo de Falha } \\
\text { Detectada }\end{array}$ & Tipo de aplicação \\
\hline HAProxy & $\begin{array}{l}\text { Auto cura, } \\
\text { migração } \\
\text { de trabalho, } \\
\text { replicação }\end{array}$ & $\begin{array}{l}\text { Reativa/ } \\
\text { Proativa }\end{array}$ & Java & $\begin{array}{l}\text { Máquina } \\
\text { Virtual }\end{array}$ & $\begin{array}{l}\text { Processo/ } \\
\text { Virtualização }\end{array}$ & $\begin{array}{l}\text { Balanceamento } \\
\text { de carga e tolerância } \\
\text { a falhas }\end{array}$ \\
\hline SHelp & $\begin{array}{l}\text { Checkpoint } \\
\text { Assure }\end{array}$ & $\begin{array}{l}\text { Reativa } \\
\text { repetir, } \\
\text { auto cura }\end{array}$ & $\begin{array}{l}\text { SQLeativa/ } \\
\text { Java }\end{array}$ & $\begin{array}{l}\text { Máquina } \\
\text { Virtual }\end{array}$ & Falha no processo & Tolerância a falhas \\
\hline Hadoop & $\begin{array}{l}\text { Migração } \\
\text { de trabalho, } \\
\text { Sguard }\end{array}$ & $\begin{array}{l}\text { Reativa/ } \\
\text { Proativa }\end{array}$ & $\begin{array}{l}\text { Java, } \\
\text { HTML, } \\
\text { CSS }\end{array}$ & Nuvem & Comunicação & Tolerância a falhas \\
\hline $\begin{array}{l}\text { Amazon } \\
\text { EC2 }\end{array}$ & $\begin{array}{l}\text { Replicação, } \\
\text { Sguard, } \\
\text { resubmissão } \\
\text { de tarefa }\end{array}$ & $\begin{array}{l}\text { Reativa/ } \\
\text { Proativa }\end{array}$ & $\begin{array}{l}\text { Amazon } \\
\text { Machine } \\
\text { Image }\end{array}$ & Nuvem & $\begin{array}{l}\text { Processo/ } \\
\text { Virtualização }\end{array}$ & $\begin{array}{l}\text { Dados } \\
\text { intensivos }\end{array}$ \\
\hline
\end{tabular}

\section{Categorização baseada em Revisão Sistemática da Literatura}

A categorização de técnicas de tolerância a falhas será feita a partir da aplicação de Revisão Sistemática da Literatura (RSL). Para RSL utilizou-se as bibliotecas digitais do IEEE Xplore, ACM Digital Library e ScienceDirect. O período considerado na busca foi de 2010 a 2017, aplicando-se restrições diferentes para cada base, com relação ao tipo de veículo (livros, conferências, periódicos). Para extrair os dados das bibliotecas criou-se strings de busca com base nas seguintes palavras chave: fault, tolerance, defect, high, 
availability, FT, OpenNebula, CloudStack, OpenStack, Cloud, Computing. Com essas palavras chave foram criadas 24 strings, sendo que quatro delas retornaram os resultados mais significativos nas três bibliotecas.

Tabela 2. Total de artigos obtidos com cada string escolhida

\begin{tabular}{|l|l|c|}
\hline String de busca & String & N $^{\circ}$ de artigos obtidos \\
\hline fault AND tolerance AND OpenStack & SS3 & 120 \\
\hline ('Fault tolerance"OR FT) AND "Cloud Computing" & SG5 & 71 \\
\hline fault AND tolerance AND OpenNebula & SN3 & 55 \\
\hline fault AND tolerance AND CloudStack & SCS3 & 10 \\
\hline \multicolumn{2}{|c|}{ Total } & 256 \\
\hline
\end{tabular}

A Tabela 2 mostra as strings selecionadas e seus respectivos resultados. Obteve-se no total 256 artigos, dos quais 39 são duplicados (15\%), levando à análise de 217 artigos para categorização. Na primeira análise desses artigos ainda foi possível identificar 48 deles como não tendo relação com o tema desejado (boa parte deles tratando de eficiência energética na nuvem).

A primeira classificação dos trabalhos envolvendo tolerância a falhas em nuvem buscou identificar para que tipo de falha uma dada solução foi proposta, nas categorias vistas na Figura 1. Nessa análise se verificou que alguns abordam mais de um tipo de falha, o que não é uma surpresa. Considerando-se agora cada tipo de falha, tem-se que 79 artigos estão relacionados a falhas de processo, 84 a falhas de comunicação, 70 a falhas de dados (armazenamento), 62 a falhas de virtualização, 6 deles não foi possível fazer a identificação, e 48 foram desclassificados por falta de afinidade com o assunto.

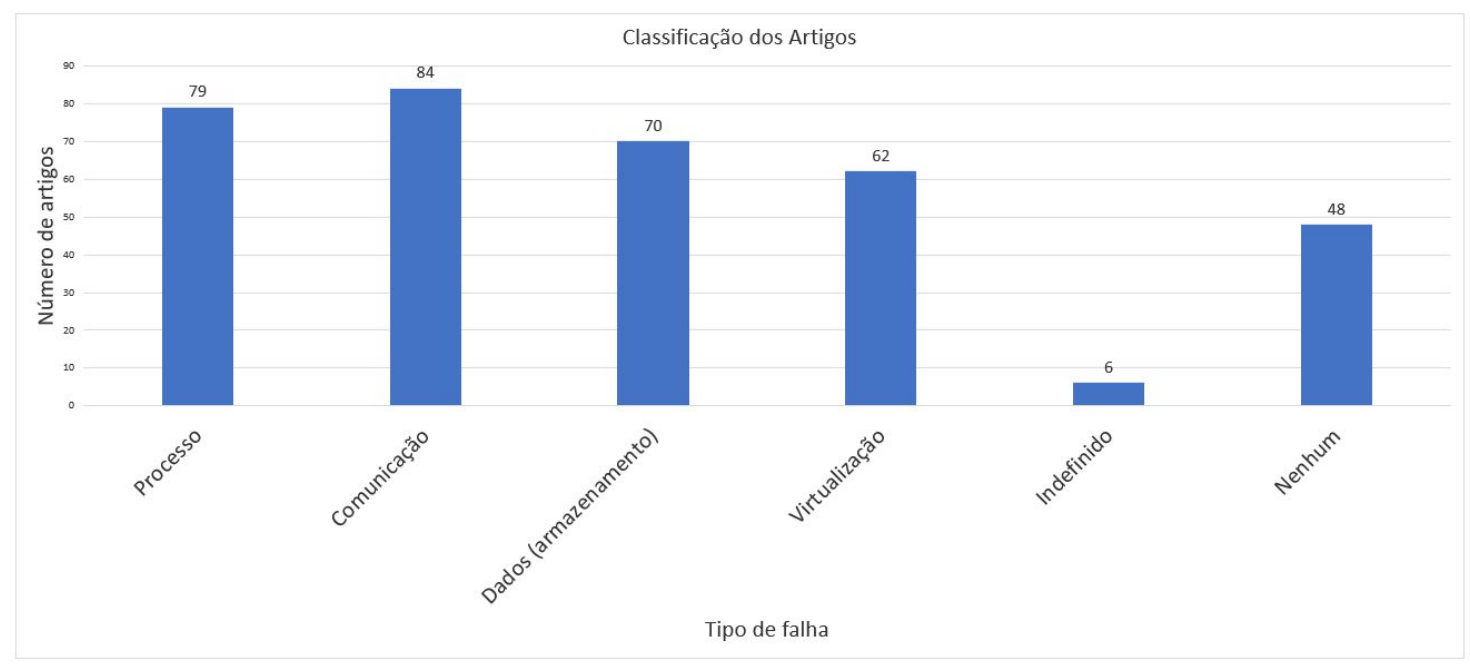

Figura 1. Quantidade de artigos publicados segundo origem da falha.

O próximo passo envolve a categorização por técnicas de tolerância a falha utilizada. A ideia é mapear quais técnicas são utilizadas com determinada falha, identificando ainda a qualidade dos resultados obtidos com essa utilização. Na Figura 2 são apresentados os resultados preliminares desse procedimento, quando aplicado para uma amostra de 20 artigos, dentre os 59 que tratam falhas de virtualização. Apesar de ser um resultado parcial é possível constatar que a técnica de migração de trabalho é a mais utilizada, 
observando-se mais uma vez que alguns artigos apresentam mais de uma técnica para tratamento de falha de virtualização.

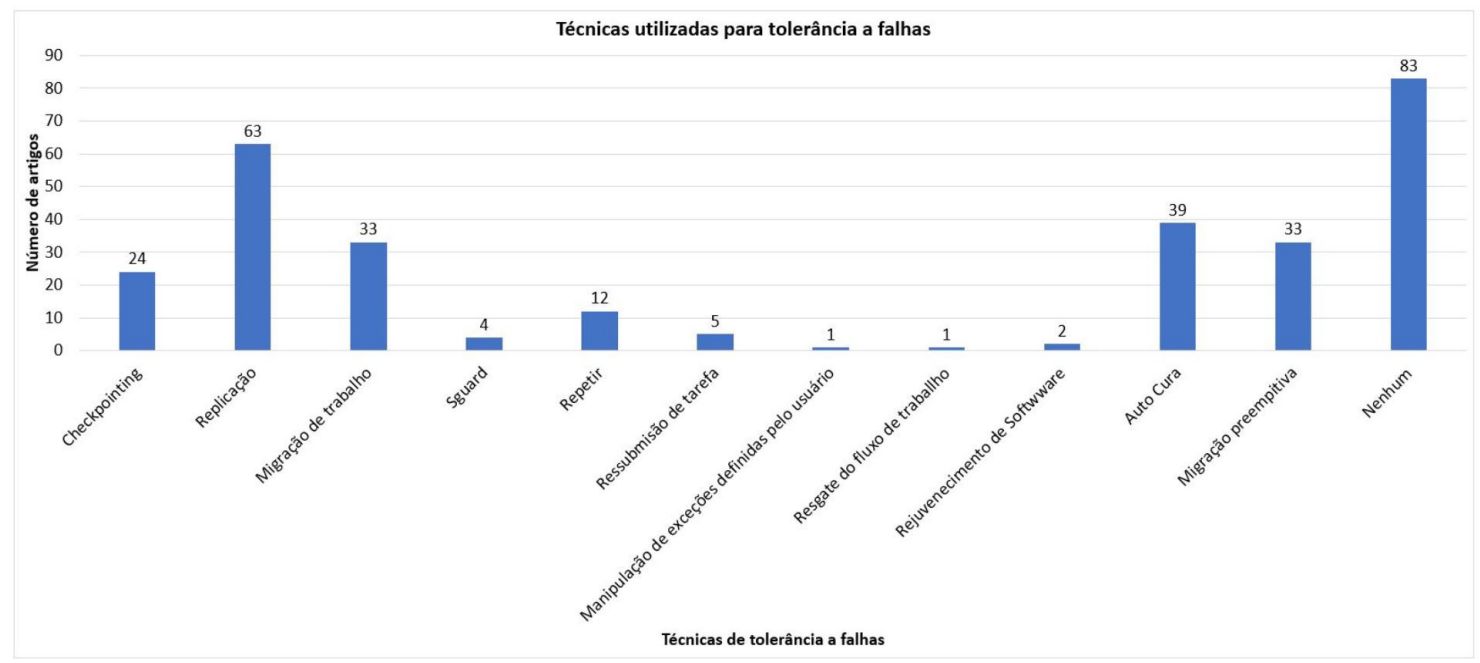

Figura 2. Técnicas utilizadas para tolerância a falhas.

\section{Considerações finais}

Por meio da categorização feita até aqui é possível concluir que existe um quantidade considerável de trabalhos relacionados a tolerância a falhas em computação em nuvem, sendo que boa parte deles trata de falhas de comunicação, ou processo ou dados. Conclui-se também que a técnica mais utilizada para se tratar falhas é replicação, possivelmente por conta de sua simplicidade e das inúmeras possibilidades de variação em sua implementação.

A sequência deste trabalho envolve a análise aprofundada dos artigos e propostas encontradas, seguida de elaboração de uma sistemática para classificar e, quando possível, qualificar/comparar os métodos de tratamento de falhas. Com isso será possível preparar um ambiente de recomendação que utilize as informações analisadas.

\section{Referências}

Bala, A. and Chana, I. (2012). Fault tolerance-challenges, techniques and implementation in cloud computing. IJCSI International Journal of Computer Science Issues, 9(1):1694-0814.

Buyya, R., Broberg, J., and Goscinski, A. (2011). Cloud Computing: Principles and Paradigms. Wiley.

Buyya, R., Yeo, C. S., Venugopal, S., Broberg, J., and Brandic, I. (2009). Cloud computing and emerging it platforms: Vision, hype, and reality for delivering computing as the 5th utility. Future Generation computer systems, 25(6):599-616.

Tanenbaum, A. S. and Van Steen, M. (2007). Distributed systems: principles and paradigms. Prentice-Hall. 